\title{
Métodos microscópicos para la cuantificación del contenido en fase vítrea de escorias siderúrgicas
}

\author{
A. RODRÍGUEZ REY, L. M. SUÁREZ del RÍO, L. CALLEJA ESCUDERO y V. G. RUIZ DE ARGANDOÑA \\ Dpto. de Geológia. Grupo de Petrofisica. Universidad deOviedo
}

ESPAN̄A

Fecha de recepción:3-X-96

Fecha de aceptación: 22-XI-96

\section{RESUMEN}

En este trabajo se revisan algunos métodos de microscopia que pueden ser utilizados para la determinación del porcentaje de particulas vitreas de escorias de alto horno. Se tratan aspectos relacionados con la preparación de muestras y se describe brevemente el procedimiento operatorio. También se comentan las principales ventajas e inconvenientes de cada método. Todos ellos son adecuados para el control de las escorias que vayan a ser utilizadas como adiciones activas en la fabricación de cementos, dado que son métodos sencillos, rápidos y económicos.

\section{SUMMARY}

Some microscopy methods that can be applied to the quantification of the percentage of vitreous particles of blast furnace slags are reviewed. Details of sample preparation are included and the experimental procedure is briefly described. The main advantages and inconveniences of each method are also considered. All of these methods are appropriate for the routine control of slags that can be used as a cement addition, since they are simple, fast and inexpensive methods.

\section{INTRODUCCIÓN}

Las escorias siderúrgicas o de alto horno constituyen un tipo de adiciones activas muy utilizadas para la fabricación de cementos, dada su capacidad hidráulica potencial. En España, el Pliego RC-93 (1) y la Norma UNE 80-301 (2) definen varios tipos de cementos que contienen escorias siderúrgicas: cemento Portland compuesto (tipo II), cemento Portland con escoria(tipo II-S), los denominados cementos de horno alto (tipos III-1 y III-2) y el cemento mixto (tipo V). En algunos casos, como el del cemento de horno alto tipo III-2, el porcentaje ponderal de escorias puede, incluso, cuadruplicar el de clínker.

Estos cementos con escorias, ya sean siderúrgicos o Portland con escorias, tienen ciertas propiedades específicas que los diferencian de los otros tipos de cementos como, por ejemplo, mayor resistencia a los sulfatos, al agua de mar, menor calor de hidratación, menores problemas de reacciones árido-álcalis, etc., que los hacen más idóneos para determinados usos.

Para poder ser usadas como adiciones activas, las escorias cle alto horno deben tener una cierta actividad hidráulica latente y, por lo tanto, unos valores adecuados de algunas características como son la composición química, el contenido en fase vítrea, la finura, etc.

Es importante, entonces, la cuantificación de estas propiedades para determinar si las escorias poseen una actividad hidráulica potencial adecuada. Los métodos de cuantificación dependen de las propiedades a determinar, si bien para algunas de ellas pueden usarse distintos métodos. 
En este trabajo se comentan algunos métodos microscópicos que pueden ser utilizados para la determinación de una de estas características de las escorias, concretamente su contenido en fase vítrea o grado de cristalinidad.

\section{CARACTERÍSTICAS PRINCIPALES DE LAS ESCORIAS DE ALTO HORNO}

Las escorias de alto horno se originan como resultado de la fusión y la combinación, a temperaturas del orden de $1.600^{\circ} \mathrm{C}$, de los constituyentes arcillosos del mineral de hierro, las cenizas del coque y la cal y la magnesia procedentes de las rocas carbonatadas usadas como fundentes en el proceso siderúrgico. Tras el posterior enfriamiento desde $1.400^{\circ} \mathrm{C}$ se obtienen las escorias.

Este fundido formado a alta temperatura tiene un elevado contenido en energía térmica y, si se enfría lentamente, da lugar a un sistema de fases cristalinas formado por silicoaluminatos de calcio y magnesio que no tienen propiedades hidráulicas (por ejemplo, minerales como la melilita o la merwinita). Pero si el enfriamiento es lo suficientemente rápido, la escoria solidifica como material vítreo que posee una hidraulicidad latente que, aunque se desarrolla con extremada lentitud, puede ser activada química, térmica o mecánicamente. Esta característica permite que la escoria pueda ser utilizada como adición activa en la fabricación de cementos. Por ello, en la industria se siguen distintos procesos de granulación o peletización que originan un rápido enfriamiento de la escoria. El porcentaje de fase vítrea, después de estos procesos, es bastante elevado y puede alcanzar el $95 \%$ ponderal.

Además, la hidraulicidad de las escorias está relacionada con otras características: composición química, finura de molido, tipo de activación, etc.

La influencia de estas características ha sido estudiada por diversos autores. Así, se han propuesto varios índices y módulos para evaluar el comportamiento hidráulico de las escorias a partir de su composición química. Puede encontrarse una revisión muy completa y actual del estado del conocimiento sobre la composición y el comportamiento hidráulico de las escorias en el trabajo de Puertas (3). Esta misma autora ha estudiado con detalle las características químicas y mineralógicas de las escorias de horno alto españolas (4).

\section{MÉTODOS DE ESTUDIO DEL GRADO DE CRISTALINIDAD}

Como el contenido de fase vítrea constituye una de las características más importantes de las escorias en lo que se refiere a su potencial hidráulico, se han dictado normas donde se establecen los contenidos mínimos. En España, la Norma UNE 80-302 (5) especifica que las escorias de horno alto "deben estar constituidas, al menos, en dos tercios de su masa, por fase vítrea".

Para la determinación del grado de cristalinidad de las escorias pueden utilizarse métodos muy diversos: quími$\cos$, de rayos $\mathrm{X}$, análisis térmico diferencial, microscópicos, etc.

Por ejemplo, Blondiau (6) determina el "grado de vitrificación" como la diferencia entre el calor de disolución, en un solvente de ácido nítricoy deácidofluorhídrico, de la escoria granulada, y el de la misma escoria después del calentamiento durante 4 horas a $1.000^{\circ} \mathrm{C}$; ambas muestras deben ensayarse después de ser molidas hasta una superficie específica de $2.700 \mathrm{~cm}^{2} / \mathrm{g}$ (Wagner). Según este autor, un grado de vitrificación comprendido entre 70 y $80 \mathrm{cal} / \mathrm{g}$, junto con ciertos valores de módulos de composición química, corresponde a escorias de buena actividad hidráulica.

También es frecuente el uso de la difracción de rayos $\mathrm{X}$ para la determinación del grado de cristalinidad de las escorias. De hecho en algunos países se han dictado normas que regulan este método, como por ejemplo la norma inglesa BS-6699: 1992 (7).

La microscopía representa otra posibilidad para la cuantificación del contenido en fase vítrea de las escorias. Fundamentalmente se trata de técnicas de microscopía óptica, aunque también se han realizado estudios con otros tipos de microscopía, como la electrónica. A continuación se comentan con cierto detalle algunas modalidades que pueden ser utilizadas.

\section{TÉCNICAS MICROSCÓPICAS}

Las técnicas microscópicas constituyen métodos sencillos, rápidos y económicos para la determinación del grado de cristalinidad de las escorias. La preparación de muestras también es rápida, sencilla y económica; asimismo, en poco tiempo puede adquirirse la destreza suficiente para llevar a cabo estudios con resultados fiables.

\subsection{Preparación de muestras}

Las muestras de escorias que se estudian en la mayor parte de las técnicas de microscopía son preparaciones en polvo. Para su elaboración, la escoria se cuartea y se muele y, tras el tamizado, se escoge una cierta fracción granulométrica que puede variar ligeramente según los distintos autores, como se detalla más adelante.

Con esta fracción granulométrica se elabora una preparación que sea una muestra representativa y uniformemente 
dispersa del polvo. Para ello se pueden usar distintos procedimientos pero el resultado final depende, sobre todo, de la destreza del operador más que del procedimiento en sí.

Las preparaciones en polvo pueden ser permanentes o no. En el caso de que no se necesite conservarlas, el procedimiento es sencillo: se echa una pequeña cantidad de polvo con una espátula en un portaobjetos de vidrio y se añade una gota de líquido de inmersión (algunas personas dispersan posteriormente el polvo en el líquido con ayuda de una espátula o una varilla de vidrio); después se coloca un cubreobjetos. Otra posibilidad es colocar el cubre sobre el polvo esparcidoen el porta y añadir líquido de inmersión en un borde del cubre de manera que el líquido vaya penetrando por capilaridad. En cualquier caso hay que evitar echar demasiada cantidad de polvo para que no se apelmace ni se formen grumos; lo ideal es que las partículas estén separadas unas de otras de manera que no se superpongan y dificulten su observación.

El líquido de inmersión debe tener un índice de refracción adecuado. Lea (8) indica que el índice de refracción de las escorias varía normalmente entre 1,635 y 1,67 , por lo que el índice de refracción del líquido de inmersión debe ser inferior a 1,635. El aceite de inmersión de índice 1,515, frecuentemente utilizado en los estudios de mineralogía óptica, da buenos resultados y permite una adecuada discriminación de las partículas de escoria. Otros autores (9) mencionan otros líquidos de inmersión como el etilenglicol o el aceite de alcanfor.

Orr y Dallevalle (10) describen un procedimiento para elaborar preparaciones permanentes de muestras en polvo que puede ser aplicado a escorias: se introduce una muestra representativa del polvo en un frasco de $10 \mathrm{ml}$, se añaden 2 ó $3 \mathrm{ml}$ de una solución al $2 \%$ de colodión en acetato de butilo, se agita enérgicamente, y se deposita una gota de la suspensión en la superficie de una lámina de agua. La película que se forma por evaporación de la gota se recoge con cuidado en un porta donde, tras secarse por completo, queda lista para su observación.

\subsection{Métodos utilizados}

\section{a) Microscopia por luz transmitida}

Nurse (11) describe este método: unos cuantos mg de fragmentos molidos de escoria de granulometría comprendida entre los tamices 300 y $170 \mathrm{BS}$ (que corresponden a 53 y a $90 \mu \mathrm{m}$ de luz de malla, respectivamente) se colocan en un porta con bromoformo y se observa bajo luz transmitida. Unos 200 aumentos son suficientes. La muestra puede prepararse también embutiéndola en una resina apropiada.

Como con todas las preparaciones de material en polvo, 10 mejor es observarlas de manera sistemática, desplazando la preparación en líneas rectas paralelas igualmente espaciadas, utilizando para ello el sistema de desplazamiento de las preparaciones del que vienen provistos los microscopios. Se consideran entonces las partículas que son intersectadas por ese sistema virtual de líneas paralelas.

\section{Se cuentan:}

- el número total de partículas.

- el número de partículas cristalinas, que aparecen coloreadas de marrón claro u oscuro.

A partir de estos datos se calcula el porcentaje de partículas vítreas, o sea el contenido en fase vítrea de la escoria.

El problema con este método es que el color no siempre es un criterio definitivo para determinar si una partícula de escoria es o no vítrea. De hecho, algunas partículas incoloras son cristalinas, como se puede comprobar utilizando alguno de los otros métodos propuestos a continuación y por lo tanto, dependiendo del tipo de escoria, los resultados pueden no ser totalmente fiables. La ventaja es que puede utilizarse con cualquier microscopio óptico. Pero si se dispone de un microscopio de polarización (petrográfico) es preferible utilizar el método que se describe a continuación.

En cualquier caso, aunque el método tal como se describe utiliza el bromoformo como líquido de inmersión, es preferible utilizar aceite de inmersión dados los problemas que podría presentar el bromoformo al ser una sustancia tóxica por inhalación.

\section{b) Microscopia de polarización por luz transmitida}

Con este método se pueden diferenciar de manera rápida y concluyente los materiales vítreos de los cristalinos. Pueden utilizarse el mismo tipo de preparaciones que para el método anterior. No obstante Hooton y Emery (9), que describen un procedimiento basado en esta técnica y que denominan método McMaster modificado, recomiendan muestras en polvo tamizadas en húmedoy de granulometría comprendida entre 45 y $63 \mu \mathrm{m}$. Este rango de tamaño de partículas es más apropiado que el descrito por Nurse (11), ya que las partículas son más pequeñas y la diferencia de tamaño entre ellas más reducida.

Las partículas vítreas son ópticamente isótropas y cuando se observan en este tipo de microscopios en la modalidad de nicoles (polarizadores) cruzados, aparecen completamente negras aunque se gire la platina del microscopio. Por el contrario, las partículas cristalinas de las escorias son ópticamente anisótropas, por lo que al ser observadas entre nicoles cruzados presentan lo que se denomina colores de interferencia, que permiten diferenciarlas fácilmente de las vítreas. La foto la es una imagen tomada en 
la modalidad de nicoles paralelos (no cruzados) en la que pueden verse las partículas molidas de escoria con la típica fractura concoidea, algunas de ellas incoloras y otras más o menos oscuras. Cuando se cruzan nicoles (foto $1 \mathrm{~b}$ ), las partículas vítreas permanecen negras y no se distinguen del fondo, también isótropo; la única partícula cristalina de este campo muestra color de interferencia, lo que permite distinguirla de las vítreas.

El color de interferencia de una partícula ópticamente anisótropa depende, además de su composición, de su orientación y de su tamaño. Las partículas cristalinas de escorias de los tamaños mencionados muestran típicamente colores de interferenciablancos, amarillos, y azules o verdes intensos. Las partículas anisótropas transparentes tienden a presentar colores de interferencia amarillos, mientras que las partículas de color marrón suelen presentar colores más vivos (azules o verdes) que no se distribuyen uniformemente, de modo que la partícula observada entre nicoles cruzados presenta zonas de distinta coloración. Algunas partículas más problemáticas, apenas transparentes e incluso casi opacas, pueden presentar una birrefringencia muy débil de aspecto lechoso. Según Hooton y Emery (9) deben considerarse cristalinas aunque sean opacas y muy débilmente anisótropas; en este criterio consiste, precisamente, la modificación del método McMaster original.

El procedimiento de contaje más sencillo consiste en examinar la muestra entre nicoles cruzados y contar, por una parte, las partículas que presentan colores de interferencia, sea cual sea su color o su intensidad (partículas cristalinas) y, por otra, las que permanecen siempre negras (las vítreas). A partir de estos datos se calcula el porcentaje de partículas vítreas, o sea, el contenido en fase vítrea.

En el método McMaster, tanto en el original como en el modificado, el procedimiento de contaje es más delicado, ya que se debe estimar el contenido de fase vítrea de cada partícula, con una "precisión" del 10\%. No obstante, en la práctica, esto es necesario sólo en el caso de partículas "mixtas"

Un inconveniente de este método es que, en el caso de preparaciones en polvo, como el aceite de inmersión también es isótropo, aparece negro entre nicoles cruzados. Por ello no es fácil diferenciar las partículas vítreas del aceite (foto $1 \mathrm{~b}$ ), y hay que estar constantemente cruzando y descruzando nicoles, lo que hace que el método pueda resultar más pesado y lento.

Para obviar este problema se puede recurrir a utilizar una lámina auxiliar y mantener los nicoles siempre cruzados. La lámina puede ser de yeso, de un espesor tal que produce un retardo de $550 \mathrm{~nm}$. Esta lámina suele venir como accesorio en los microscopios petrográficos. Cuando se observa la preparación entre nicoles cruzados y con la lámina auxiliar insertada en el microscopio, las partículas vítreas aparecen de color rosa, igual que el aceite de inmersión, aunque con los bordes claramente resaltados,

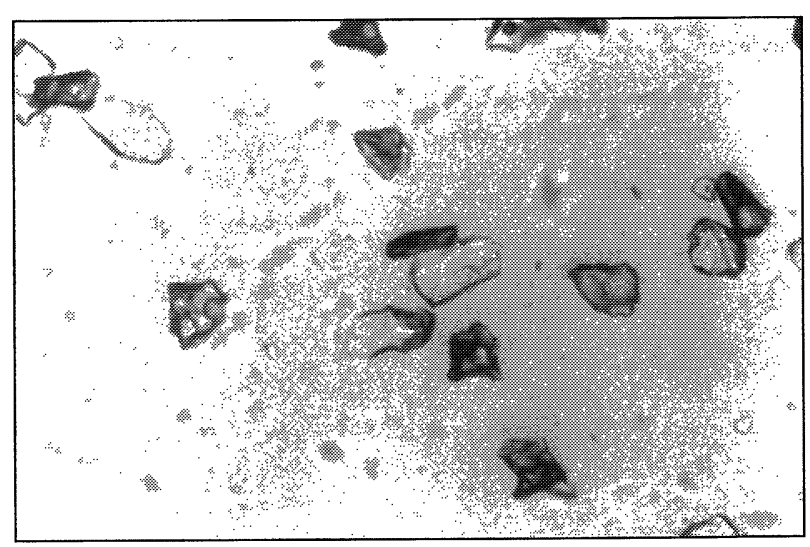

1a

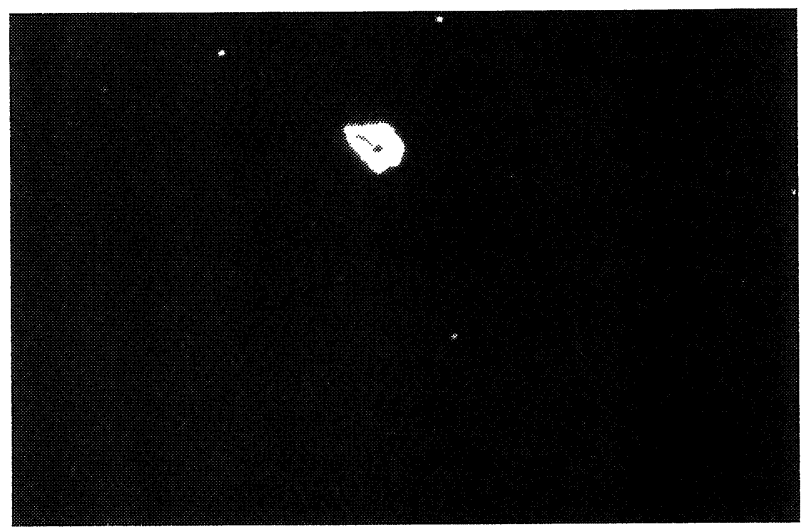

$1 b$

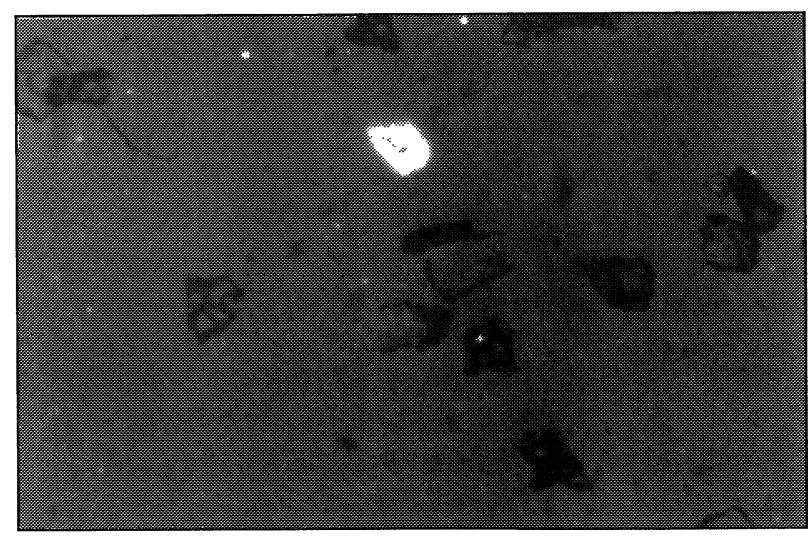

$1 \mathrm{c}$

Foto 1.- Muestra en polvo de escoria siderúrgica observada mediante microscopia óptica de polarización por luz transmitida: 1a) nicoles paralelos; 1b) nicoles cruzados; 1c) nicoles cruzados con lámina auxiliar de yeso. $(1 \mathrm{~cm}=110 \mu \mathrm{m})$

Photo 1.- Powder sample of blast furnace slag under transmitted-light polarizing microscopy: I a) plane polarized light; lb) crossed nicols; ; c) crossed nicols with gypsum auxiliary plate. $(1 \mathrm{~cm}=110 \mu \mathrm{m})$. 
a diferencia de lo que ocurre cuando se observa la preparación sin la lámina de yeso; por su parte, las partículas cristalinas presentan color de interferencia modificado por la inserción de la lámina que cambia de color si se gira la platina del microscopio. En la foto 1c, que es una imagen del mismo campo que el de las fotos $1 \mathrm{a}$ y $1 \mathrm{~b}$, tomada con nicoles cruzados y lámina de yeso, se comprueba la utilidad de esta lámina: además de verse el color de interferencia de la partícula cristalina, las partículas vítreas destacan claramente del fondo.

Otra precaución que también hay que tener en cuenta es que algunas partículas vítreas pueden presentar birrefringencia debida a tensiones, lo que las hace difíciles de distinguir de las partículas verdaderamente cristalinas. Además, algunas partículas cristalinas, cuando se observan en una posición determınada (perpendicularmente a algún eje óptico) aparecen como isótropas y pueden ser confundidas con las vitreas. De todas formas, los errores de cuantificación que se pueden derivar de estos hechos pueden, en la práctica, ser considerados como despreciables.

Por su sencillez y su fiabilidad, la microscopía óptica de polarización por luz transmitida es un método muy recomendable para la cuantificación del grado de cristalinidad de las escorias.

La explicación detallada de los fenómenos de microscopía de polarización y sus fundamentos, así como recomendaciones para la puesta a punto y el manejo del microscopio petrográfico, puede encontrarse en muchos de los tratados existentes de Cristalografía Optica, como, por ejemplo, en Bloss (12)

\section{c) Microscopia de fluorescencia}

Este método ha sido usado frecuentemente en Alemania y aparece citado por Kramer (13). La muestra se observa con luz ultravioleta. Las particulas vitreas emiten fluorescencia roja o rosa, mientras las partículas muy cristalinas emiten fluorescencia azul o violeta. Incluso se habla de la idoneidad de esta técnica para distinguir entre material vítreo de distinta actividad hidráulica; sin embargo, Lea(8) comenta que la experiencia con escorias de Gran Bretaña indica que esta posibilidad adicional no se puede generalizar y sólo es recomendable para escorias producidas por un determinado proceso, e incluso procedentes del mismo horno.

\section{d) Microscopia por luz reflejada}

En este caso se utilizan preparaciones pulidas de escorias que han de ser atacadas previamente con una disolución de ácido nítrico en etanol; las partículas vítreas se colorean de marrón tras el ataque, mientras que las fases cristalinas adquieren distintos colores según su composición mineralógica: azul la merwinita e incolora la melilita, (14).

\subsection{Métodos de microscopía electrónica}

Dadas sus posibilidades, la microscopía electrónica de barrido también puede emplearse con buenos resultados en el estudio de las escorias, especialmente si se complementa con la espectroscopía de rayos $\mathrm{X}$ mediante microsonda electrónica. Sersale (15) cita el trabajo de Demoulian et al. (16) en el que estudian las características de los cristales de merwinita y de melilita y la distribución de los distintos elementos químicos en los mismos. El potasio y el azufre se localizan preferentemente en la fase vítrea, y la distribución del aluminio y del magnesio en la fase vítrea es distinta que en las fases cristalinas; asimismo, se observa que los cristales presentan una zonación composicional, con unos núcleos más ricos en akermanita que su periferia.

\subsection{Cálculo de errores}

Los errores implicados en esta metodología pueden clasificarse en experimentales (algunos de los cuales ya han sido comentados anteriormente) y de recuento.

En cuanto a estos últimos hay que resaltar que, en los métodos propuestos, el resultado que se obtiene es el porcentaje de partículas vítreas, o sea, un porcentaje volumétrico, y no el porcentaje ponderal de fase vítrea que es el que aparece en las normas y el que se obtiene por otros métodos. En todo caso, se puede hacer una estimación de dicho porcentaje si se tienen datos de la densidad de los materiales vítreos y de los minerales y si se considera que todas las particulas son prácticamente del mismo tamaño. Estas consideraciones son fundamentales cuando se comparan los valores del grado de cristalinidad obtenidos por métodos microscópicos con los proporcionados por otras técnicas como la difracción de rayos X. Hooton y Emery (9) señalan, de todas formas, que los resultados obtenidos por el método McMaster, de microscopía óptica de polarización, son comparables a los que se obtienen mediante difracción de rayos $\mathrm{X}$

Como en todos los procedimientos de recuento de puntos, el error máximo depende del porcentaje de la fase en cuestión y, sobre todo, del número total de puntos (en este caso partículas) que se cuenten, de manera que, a mayor número de partículas contadas, menor error.

Para estimar los errores de recuento en el caso de contaje de puntos o de partículas se suele considerar una distribución binomial.

La desviación estándar (s) de una distribución binomial se calcula mediante la siguiente expresión:

$$
s=\sqrt{\frac{p \cdot q}{N}}
$$


donde:

p: porcentaje de partículas vítreas (en tanto por uno).

q: $(1-p)$.

$\mathrm{N}$ : número total de partículas contadas.

el error absoluto ( $\varepsilon$ ) correspondiente a un intervalo de confianza del $95 \%$ es:

$$
\varepsilon= \pm 2 \mathrm{~s}
$$

y el error relativo (E):

$$
E=\varepsilon / p
$$

Estas expresiones sirven también para estimar el número de partículas que hay que contar para no sobrepasar un determinado error para un intervalo de confianza dado.

Hooton y Emery (9) proponen otra alternativa: se cuentan 3 lotes de 50 partículas cada uno y se calcula el porcentaje de fase vítrea de cada uno de ellos. El porcentaje de fase vítrea de la escoria se halla haciendo la media de los porcentajes de fase vítrea de los lotes, y se calcula, además, el coeficiente de variación. Mientras que este número de partículas contadas (150) puede ser suficiente para el control rutinario de escorias muy vítreas, con un porcentaje de fase vítrea del orden del $95 \%$ (en cuyo caso el error absoluto sería de $\pm 3,6$ ), puede resultar escaso en estudios comparativos detallados de escorias, especialmente cuan- do el porcentaje en fase vítrea de las mismas no llega al $80 \%$ (error absoluto $\pm 6,5$ ).

\section{CONCLUSIONES}

Las técnicas microscópicas son métodos muy indicados para la cuantificación del porcentaje de fase vítrea (grado de cristalinidad) de las escorias siderúrgicas. Sus principales ventajas radican en su sencillez, rapidez y bajo coste. Además las muestras que se utilizan son rápidas y fáciles de preparar, y su coste es mínimo. Con el uso de estas técnicas microscópicas se puede disponer con rapidez de datos muy importantes para caracterizar la idoneidad de una escoria de horno alto para ser usada como adición activa en la fabricación de cementos. Dadas sus características (facilidad y rapidez de preparación de muestras, su sencillez y su fiabilidad) la microscopía óptica de polarización por luz transmitida representa una de las mejores opciones disponibles.

\section{AGRADECIMIENTOS}

Este trabajo ha sido realizado dentro del contrato de investigación CN-96-064-B1 entre la Universidad de Oviedo y el laboratorio Estabisol, S. A.. Los autores desean agradecer a la Dra. F. Puertas, del Instituto de Ciencias de la Construcción Eduardo Torroja, sus valiosos comentarios y sugerencias a un manuscrito previo de este trabajo.

\section{BIBLIOGRAFIA}

(1) RC-93.: Instrucción para la recepción de cementos. MOPTMA. 1993.

(2) Norma UNE 80-301/1988.: Cementos. Definiciones, clasificación y Especificaciones.

(3) PUERTAS, F.: (1993). Materiales de Construcción, Vol. 43, No. 229, 37-48.

(4) PUERTAS, F.: (1987). Tesis doctoral. Universidad Autónoma de Madrid.

(5) Norma UNE 80-302/1985.: Cementos. Especificaciones químicas para sus constituyentes.

(6) BLONDiAU, L.: (1951). Rev. Mater. Constr. 424, 6; 425, 42.

(7) BS 6699:1992.: Appendix D. Method of determining the glass content of ggbs by X-ray diffraction. British Standards Institution.

(8) LEA, F.M.: The chemistry of cement and concrete. $3^{\mathrm{a}}$ ed. Edward Arnold. Londres, 1970.

(9) HOOTON, R.E. \& EMERY, J.J.: (1983). Ist. Int. Conf. on the use of fly ash, silica fume, slag and other mineral by-products in concrete. (Montebello, Canada, July-August, 1983). Vol. II, 943-962.

(10) ORR, C. \& DALlEVALLE, J.M.: Fine particle measurement. Macmillan, Nueva York, 1959.

(11) NURSE, R.W.: "Slag Cements" (In The Chemistry of Cements, H.F.W.Taylor, (ed.). Academic Press. Londres, 1964.

(12) BLOSS, F.D.: Introducción a los métodos de Cristalografía Optica. Omega. Barcelona, 1970. 
(13) KRAMER, W.: (1962). Proc. 4th Int. Symp. Chem. Cement (Washington 1960), 957.

(14) FREARSON, J.P.J.; SIMS, I. \& UREN, J.M.: (1986). 2nd Int. Conf. Concr., Madrid, 21-25.

(15) SERSALE, R.: "Aspects of the chemistry of additions" (In Advances in Cement Technology, S.N. Gosh, ed. Pergamon Press. Oxford, 1983).

(16) DEMOULIAN, E.; GOURDIN, P.; HAWTHORN, F. \& VERNET, C.: (1980). 7th Int. C. Chem. Cem., Paris, II, III-89.

\section{Publicaciones del Instituto Eduardo Torroja-CSIC}

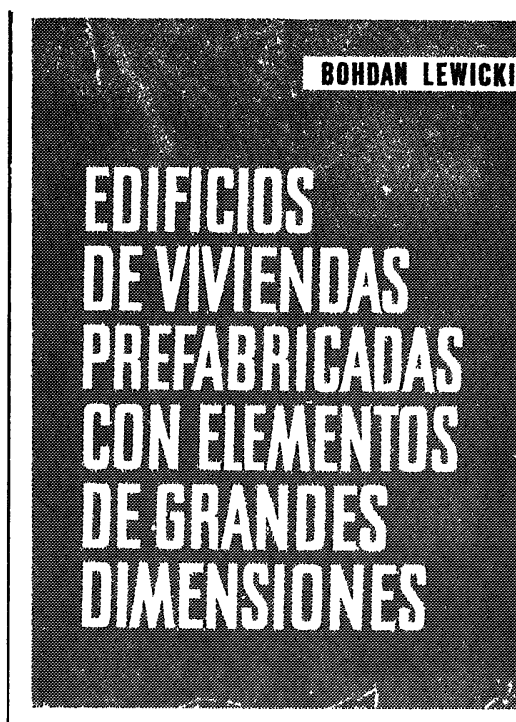

Bohdan Lewicki

Este libro trata de los problemas relativos a la construcción de los edificios de viviendas o públicos realizados con elementos prefabricados de grandes dimensiones. Se han estudiado los problemas de arriostramiento, asi como los que plantea la resistencia de los elementos y de la estructura; se han examinado las cuestiones de orden higrotérmico, acústico y de resistencia al fuego; también se ha profundizado en el estudio de la estanquidad de los muros exteriores y de las juntas.

La obra incluye numerosas ilustraciones que dan detalles de diversas soluciones, asi como ejemplos de cálculo, tablas de valores numéricos, diagramas y ábacos.

Un volumen encuadernado en tela, de $24 \times 17 \cdot \mathrm{cm}$, compuesto de 616 págs.

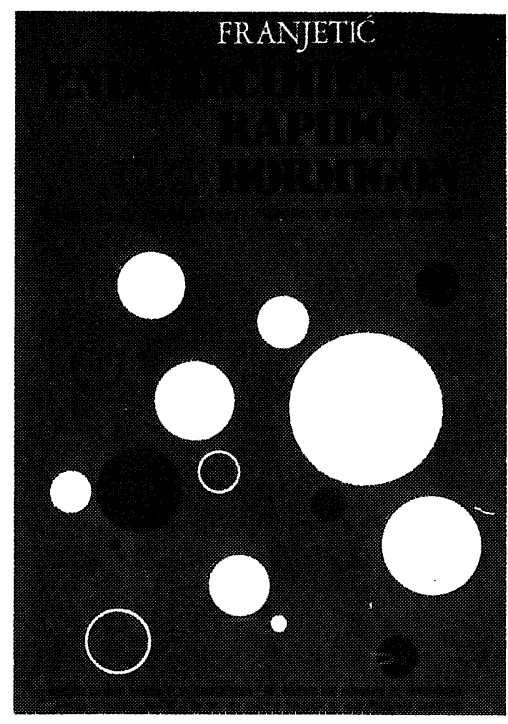

Zorislav Franjetić

En la obra de Franjetić se expone de una forma minuciosa, ordenada y sistemática, todo un cuerpo de doctrina que reúne el conocimiento actual sobre el endurecimiento rápido del hormigón. Parte el autor de los principios básicos y llega a las últimas conprincipios básicos y llega a las últimas connómicas.

Es una obra de consulta, tanto para el investigador sobre la materia, como para el proyectista y el realizador y montador de plantas e instalaciones y equipos de curado y endurecimiento rápido.

Un volumen encuadernado en cartóné, de $17 \times 24,5 \mathrm{~cm}$, compuesto de 385 págs. 110 figuras y 10 tablas.

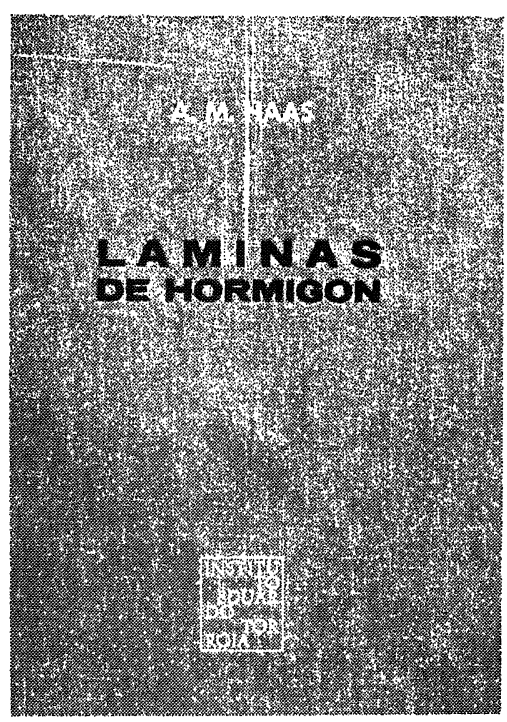

\section{A. M. Haas}

Al escribir este libro el autor intentó poner a disposición de los estudiantes y de los ingenieros unos conocimientos prácticos, adecuados para servir de guia en el diseño y construcción de láminas delgadas de hormigón.

El autor está convencido de que el éxito en el diseño de una lámina exige, por parte del proyectista, un examen de las tres fases por las que pasa la materialización de la lámina: el diseño, el análisis estructural y la construcción de la estructura.

Un volumen encuadernado en tela, de $17 \times 24,5 \mathrm{~cm}$, compuesto de 420 págs., 141 figuras, 22 fotografias y 6 tablas. 